\title{
Primary Motor and Sensory Cortex Activation during Motor Performance and Motor Imagery: A Functional Magnetic Resonance Imaging Study
}

\author{
Carlo A. Porro, ${ }^{1}$ Maria Pia Francescato, ${ }^{1}$ Valentina Cettolo, ${ }^{2}$ Mathew E. Diamond, ${ }^{1}$ Patrizia Baraldi, ${ }^{3}$ \\ Chiava Zuiani, ${ }^{2}$ Massimo Bazzocchi, ${ }^{2}$ and Pietro E. di Prampero ${ }^{1}$

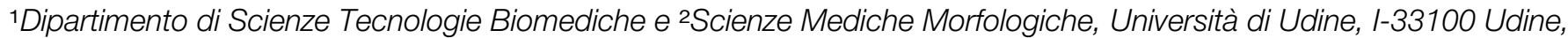 \\ Italy, and ${ }^{3}$ Dipartimento di Scienze Biomediche, Università di Modena, I-41100 Modena, Italy
}

The intensity and spatial distribution of functional activation in the left precentral and postcentral gyri during actual motor performance (MP) and mental representation [motor imagery (MI)] of self-paced finger-to-thumb opposition movements of the dominant hand were investigated in fourteen righthanded volunteers by functional magnetic resonance imaging (fMRI) techniques. Significant increases in mean normalized $\mathrm{fMRI}$ signal intensities over values obtained during the control (visual imagery) tasks were found in a region including the anterior bank and crown of the central sulcus, the presumed site of the primary motor cortex, during both MP (mean percentage increase, $2.1 \%$ ) and $\mathrm{MI}(0.8 \%)$. In the anterior portion of the precentral gyrus and the postcentral gyrus, mean functional activity levels were also increased during both conditions (MP, 1.7 and 1.2\%; MI, 0.6 and $0.4 \%$, respectively).
To locate activated foci during MI, MP, or both conditions, the time course of the signal intensities of pixels lying in the precentral or postcentral gyrus was plotted against single-step or double-step waveforms, where the steps of the waveform corresponded to different tasks. Pixels significantly $(r>0.7)$ activated during both MP and MI were identified in each region in the majority of subjects; percentage increases in signal intensity during $\mathrm{MI}$ were on average $30 \%$ as great as increases during MP. The pixels activated during both MP and Ml appear to represent a large fraction of the whole population activated during MP. These results support the hypothesis that MI and $\mathrm{MP}$ involve overlapping neural networks in perirolandic cortical areas.

Key words: primary motor cortex; primary somatosensory cortex; motor performance; motor imagery; functional magnetic resonance imaging; brain mapping
Motor imagery (MI) may be defined as the mental rehearsal of simple or complex motor acts that is not accompanied by overt body movements. Two kinds of mental representations of motor acts can be generated by normal subjects: an "internal" or firstperson process in which subjects feel themselves executing movements, and an "external" or third-person process involving a visual representation of actions (see Mahoney and Avener, 1987). Unlike visual imagery (VI), internal (or kinesthetic) MI is difficult to describe verbally. Nevertheless, it is usually characterized by vivid mental representations and by changes in heart and respiration rates that are related to the degree of mental effort (Decety et al., 1991; Wang and Morgan, 1992).

It is currently debated to what extent brain networks activated during internal MI overlap those involved in the preparation and execution of real motor acts (Jeannerod, 1994). This issue has been addressed using a variety of brain mapping techniques. Early studies investigating regional cerebral blood flow levels by single photon emission or positron emission tomography (PET) techniques demonstrated the activation during MI of different motor-

Received March 25, 1996; revised Sept. 4, 1996; accepted Sept. 9, 1996.

This work was supported by funds of Consiglio Nazionale delle Ricerche and Ministero Università Ricerca Scientifica e Tecnologica, a grant from Siemens Italia SpA to V.C., and Telethon Grant 779 to P.E.P. We thank R. Cox, Biophysics Research Institute, Medical College of Wisconsin, for providing the software package for image registration and analyses.

Correspondence should be addressed to Prof. Carlo A. Porro, Dipartimento di Scienze Tecnologie Biomediche, Universitá di Udine, Via Gervasutta 48, I-33100 Udine, Italy.

Copyright (C) 1996 Society for Neuroscience $0270-6474 / 96 / 167688-11 \$ 05.00 / 0$ related regions, such as the supplementary motor area, lateral frontal (premotor) areas, and cerebellum, but they did not provide evidence for increased activity in the primary sensorimotor cortex (Ingvar and Philipson, 1977; Roland et al., 1980; Fox et al., 1987; Decety et al., 1990).

Conflicting results have been obtained by more recent PET or functional magnetic resonance imaging (fMRI) mapping studies. Activated foci in or around the primary motor area have been described in PET studies by Lang et al. (1994) during oculomotor imagery, and by Stephan et al. (1995) during mental representation of upper extremity movements, but not during imagery of grasping movements (Decety et al., 1994) or implicit imagery of hand rotations (Parsons et al., 1995). Moreover, in the study by Stephan et al., only two of six subjects showed activated foci in or near the anterior bank of the central sulcus, the likely site of primary motor cortex (Brodmann area 4) in humans (Roland and Zilles, 1994; Geyer et al., 1995). Primary sensorimotor cortex activation during MI has been denied by two early fMRI works (Rao et al., 1993; Sanes et al., 1993), whereas it was found by other groups, at least in some subjects (Leonardo et al., 1995; Sabbah et al., 1995). These studies, however, did not provide a quantitative assessment of the relative contribution of precentral and postcentral areas.

The present study was designed to assess by high-resolution fMRI techniques whether perirolandic areas show increased functional activity levels during mental representation of sequential finger movements and to investigate the spatial distribution and intensity of activated foci during MI and overt motor behavior. 
The results suggest that overlapping neural networks in the primary motor and somatosensory areas are activated during mental representation and actual performance of motor acts.

\section{MATERIALS AND METHODS}

Fourteen right-handed volunteers (4 males, 10 females; 20-39 years of age, mean, 24.6) were studied. All gave their informed consent for the procedure, and none had a history, symptoms, or signs of neurological or psychiatric disease. Handedness was assessed using a short questionnaire based on the Edinburgh scale (Oldfield, 1971).

Data acquisition. The experiments were performed using a Magnetom SP 4000 (Siemens, Erlangen, Germany) superconducting $1.5 \mathrm{~T}$ wholebody magnetic resonance (MR) system equipped with a standard circularly polarized head coil. Head motion was minimized by an adjustable padded head holder. Field homogeneity was adjusted by a global shimming procedure for each subject, with line widths being $\sim 20 \mathrm{~Hz}$. To locate the precentral and postcentral gyri, multislice $T_{1}$-weighted spinecho sagittal, axial, and coronal images [repetition time $(T R) /$ echo time $(T E)=600 / 15 \mathrm{msec}$ ] were acquired. Two adjacent oblique (axial to sagittal) planes were then defined along the central sulcus of the left hemisphere, including the putative hand representation area of the primary sensorimotor cortex (Talairach and Tournoux, 1988; Colebatch et al., 1991; Grafton et al., 1991, 1993; Rumeau et al., 1994). The anatomy of these oblique planes was shown on $T_{1}$-weighted images [field of view $($ FOV $)=220 \mathrm{~mm} ; 256 \times 256$ matrix; slice thickness $=4 \mathrm{~mm}]$. The more superficial plane covered the gray matter at the convexity of the precentral and postcentral gyri, whereas the deeper one included the anterior and posterior banks of the central sulcus. For the activation studies, images from the same planes were acquired using a gradient-echo fast low-angle shot $(\mathrm{FLASH})$ sequence $(T R / T E=91 / 60 \mathrm{msec}$, flip angle $=$ $40^{\circ} ; 128 \times 128$ matrix; FOV $=220 \mathrm{~mm}$; slice thickness $=4 \mathrm{~mm}$ ). The FLASH images were interpolated to and displayed as a $256 \times 256$ matrix, thus yielding a pixel size of $0.86 \times 0.86 \times 4.0 \mathrm{~mm}$. Total scan time for one slice was $14 \mathrm{sec}$.

Each subject was asked to perform three different tasks sequentially during the experiments: mental representation of a stationary visual scene (VI), mental representation of self-paced, sequential finger-to-thumb opposition movements of the right hand at a frequency of $\sim 2 \mathrm{~Hz}(\mathrm{MI})$, and actual execution of the same motor performance (MP). VI was taken as a reference state to control for possible aspecific effects related to imagery. Subjects were instructed to perform the motor sequence at a constant rate, exerting a light pressure at each finger contact with the thumb. The order of finger-tapping was simply 2-3-4-5-2-3-4-5. The instructions for MI were "to imagine using the right hand to perform movements and feeling the sensations associated with finger-tapping, while keeping the hand still." For VI, subjects were asked to mentally represent a familiar landscape (typically, a natural setting). They were requested to scan the mental scene and to focus on particular objects but not to imagine themselves moving any part of the body. It was emphasized that during the MI and VI tasks, the arms had to remain still with the right hand resting on the chest and the fingers in a midflexion position.

Subjects were allowed to practice each of the three tasks during the time ( $\sim 40 \mathrm{~min})$ they were lying in the magnet, before the acquisition of functional images. All of them reported being able to execute the tasks, although some judged it quite difficult to maintain the same vividness of mental representations (MI or VI) over the whole experimental period. Hand movements were monitored throughout the experiments using a video camera. Dynamic data sets were acquired in blocks of four images, each lasting $14 \times 4=56 \mathrm{sec}$, while subjects performed one of the tasks. Subjects started the execution of the next task immediately after the end of each block. The functional sequence, however, started $12 \mathrm{sec}$ later; this delay was chosen to avoid the acquisition of data during the transition periods (Kwong et al., 1992; Bandettini et al., 1993; Friston et al., 1994).

In a first group of experiments (experiment 1), performed in 12 subjects, the task sequence was VI-MI-MP. In a second group of experiments (experiment 2), performed in eight volunteers (six of whom had already participated in experiment 1), the order of tasks was reversed, and rest periods of the same duration as the other conditions were added, during which subjects were asked to relax, keeping the arms and fingers in the same posture as during the VI and MI tasks. The task order was MP-Rest-MI-VI. In each experiment, the task sequence was repeated three times during the acquisition of functional images from each plane of interest. A total of 36 (48 for experiment 2) images was thus acquired for each plane over a total period of 20 (27 for experiment 2$)$ min.
Data analyses. Image analyses were performed using a Silicon Graphics Indy workstation. For each study and each anatomical image, three regions of interest (ROIs) were identified and manually outlined by one investigator and independently confirmed by another: the postcentral gyrus (PostCG) and the anterior (PreCGAnt) and posterior (PreCGPost) portions of the precentral gyrus. In the superficial plane, the boundary between PreCGAnt and PreCGPost was traced at approximately 1/3 the distance between the central sulcus and the precentral sulcus, which was identifiable in all subjects. In the deeper plane, the same boundary corresponded typically to the white matter underlying the precentral gyrus. Thus, PreCGPost included the anterior bank and crown of the central sulcus. PostCG was defined as the region lying posterior to the central sulcus; it was limited posteriorly by another sulcus, the shape of which, however, was quite different from one subject to another. As a first approximation, PostCG corresponds to the "hand area" of the primary somatosensory cortex (Brodmann areas 3, 1,2), PreCGPost to the "hand region" of the primary motor cortex (area 4), and PreCGAnt to the adjacent portion of the lateral premotor cortex (area 6). The number of pixels, including both planes, in the three ROIs (mean \pm SEM of 20 studies) was $782 \pm 50$ for PreCGAnt, $632 \pm 33$ for PreCGPost, and $1478 \pm 81$ for PostCG. Care was taken to exclude pixels associated with structures other than nerve tissue, such as visible blood vessels or the inside of sulci.

To correct for possible movement artifacts, functional images were aligned by a software procedure implemented by Robert Cox (AFNI software package) and based on a previously described algorithm (see Irani and Peleg, 1991). Briefly, registration was accomplished by minimizing the error functional $\mathbf{E}(u, v, h)=\Sigma_{x, y}[\mathbf{I}(x, y)-\mathbf{J}(\mathbf{T}(u, v, h)(x, y))]^{2}$, where $\mathbf{J}(x, y)$ is the "base" image, $\mathbf{I}(x, y)$ is the image to be aligned to it, $\mathbf{T}(u, v, h)$ is the transformation with shift parameters $(u, v)$ and with rotation angle $h$. After finding the $(u, v, h)$ that minimizes the error functional, $\mathbf{I}(x, y)$ was transformed with $\mathbf{T}(-u,-v,-h)$ using bicubic interpolation for resampling $\mathbf{I}$. Then the minimization was repeated; that is, simple descent gradient was used to minimize $\mathbf{E}$. The procedure was first performed with a $\mathbf{J}(x, y)$ that has been smoothed with a Gaussian filter with full-width at half-maximum (FWHM) equal to 4 pixels. After minimizing the error, the procedure was repeated with $\mathbf{J}(x, y)$ smoothed with an $\mathrm{FWHM}=1$ pixel. Mean $\pm \operatorname{SEM} u$ and $v$ values from the 20 studies, expressed in pixel fractions (unsigned), were $0.297 \pm 0.008$ (range for individual images, $0-1.846$ ) and $0.336 \pm 0.009$ (range, $0-2.222$ ), respectively; $h$ value was $0.232 \pm 0.006^{\circ}\left(\right.$ range, $\left.0-1771^{\circ}\right)$. Even if subtle artifacts caused by head motion during single-image acquisition cannot be excluded, no obvious false-positive results (e.g., activated pixels at the borders between the gray matter and surrounding tissue) were found. A preliminary analysis performed on the same set of images showed that the number of activated pixels during actual MP was not significantly different using the present registration algorithm or that described by Woods et al. (1992). In each case, the "base" functional image was acquired immediately after the $T_{1}$-weighted anatomical image of the same plane. The boundaries of the three ROIs identified on anatomical images were automatically projected onto the aligned functional images, and subsequent analyses were performed only on pixels lying inside the three ROIs.

To analyze the intensity and time profile of fMRI signal changes, data were normalized in every study and for each pixel by dividing the actual value in each image by the mean signal intensity of the same pixel in the 12 images acquired during the control (VI) condition. Mean values of signal intensity of all pixels (in the superficial and deep planes) lying within the three ROIs, or of pixel populations selected by correlation analyses (see below), were then calculated and analyzed by repeatedmeasures ANOVA, using the SPSS for Windows software package. To ascertain potential variations of task-related neural activation over time, the block order (possible levels, 1-3) and image order within a block (1-4) were added to region (PreCGAnt, PreCGPost, PostCG) and task (MP, MI, VI and rest in experiment 2) as within-subject factors. The Mauchly test of sphericity was used to test the hypothesis that the covariance matrix of the transformed variables has a constant variance on the diagonal and zeroes off the diagonal. If the sphericity assumption appeared to be violated $(p<0.05)$, the Greenhouse-Geisser $\epsilon$ was used to adjust degrees of freedom for the averaged results. Whenever firstlevel tests were significant, difference (reverse Helmert) contrasts were applied to assess differences between variable levels. A value of $p<0.05$ was assumed as the significance level.

To locate activated foci during MI and MP in individual subjects, the signal time courses in each pixel were compared with predefined, taskrelated waveforms, thus creating statistical maps based on correlation 
coefficients $(r)$, with a possible range of -1 to 1 (Bandettini et al., 1993). A correlation coefficient of 0.7 was assumed as the threshold for significance, corresponding to an approximate $p$ value of $2 \times 10^{-6}$ (two-tailed) for analyses performed on 36 images. Because the highest number of statistical comparisons in the subject displaying the largest ROIs was 11,496 ( 3832 pixels in the 3 ROIs $\times 3$ correlation analyses, see below), the adopted significance threshold yielded a Bonferroni-corrected $\alpha$ level of $<0.05$. Preliminary analyses, done on 36 images acquired from phantoms $(n=9$; mean extent of the $\mathrm{ROI} \cong 5300$ pixels $)$ or from the perirolandic cortex of healthy volunteers $(n=5$; mean extent of the ROI $\cong 1400$ pixels) during 9 min of a resting, relaxed state or repeated 3 min periods of finger-tapping at a frequency of $2 \mathrm{~Hz}$, gave no "false-positive" pixel, using any of the waveforms used for data analysis and a threshold of $r=0.7$.

Negative correlations were found for a small number of pixels in a few subjects; only positive results are reported here. Statistical maps were overlaid on the $T_{1}$-weighted anatomical images obtained at the same planes. For each subject and each ROI, the occurrence of at least 4 pixels significantly correlated with a given waveform was assumed to be a threshold of activation. To identify sites of activation using standard coordinates, the MRI scans were resized into the anatomical space of the atlas of Talairach and Tournoux (1988). The Talairach coordinates of the centers of mass of the activated pixels within each ROI were then calculated using software procedures (AFNI) developed by Robert Cox.

Electromyographic (EMG) recording. EMG recordings were not performed during the fMRI study because of technical limitations. In a separate experimental session, performed outside the MR equipment, EMG activity was recorded in each volunteer from surface electrodes overlaying the thenar eminence or flexor digitorum superficialis on the medial aspect of the forearm. Subjects performed the three tasks (VI, MI, and MP) in the same order as during the fMRI experiment 1, and each recording session lasted $\sim 10 \mathrm{~min}$. After conventional rectification and filtering of the digitized EMG data, integral values of EMG activity over successive $4 \mathrm{sec}$ periods were calculated in each subject and later analyzed by ANOVA. Although no subject made overt movements of the right arm during MI, a mild increase of EMG activity during MI relative to the control condition (VI) was observed in 4 of 14 subjects for recordings from the thenar eminence, in 1 subject from both recording sites, and in 3 of 14 subjects from the medial forearm. However, the integrated EMG data were not significantly affected by MI in the whole population at thenar $(F=0.96, p>0.4)$ or forearm $(F=0.05, p>0.8)$ sites.

\section{RESULTS}

None of the subjects displayed overt hand motion during the two imagery conditions. The rate and amplitude of hand movements during the MP task showed little intraindividual and interindividual variations, as judged independently by two investigators.

\section{Experiment 1 \\ Mean signal intensity changes in the precentral and postcentral regions}

Mean values of normalized signal intensity for individual subjects were obtained for each image from all pixels lying within each ROI: the PreCGAnt and PreCGPost parts of the precentral gyrus and the PostCG. A four-way, repeated-measures ANOVA (region $\times$ task $\times$ block $\times$ image: $3 \times 3 \times 3 \times 4$ ) showed that mean fMRI signals were differently modulated in the three regions according to task (region, $F=6.19, p<0.01$; task, $F=23.61, p<$ 0.001 ; task $\times$ region, $F=4.24, p<0.05)$. MI was associated with significantly higher values $(t=2.69, p<0.05)$ than VI. Moreover, images obtained during MP were characterized by higher values than both of the other conditions $(t=6.13, p<0.001)$. No significant difference was found for data obtained in different blocks or for different images within a block. Therefore, for each subject, data from different blocks and time points were grouped and additional one-way, repeated-measures ANOVAs were performed for each region on values representing the mean normalized fMRI signal of all images obtained during the execution of each task (VI, MI, or MP). In all regions, fMRI signals were significantly modulated by task (PreCGAnt: $F=17.13, p<0.001$;

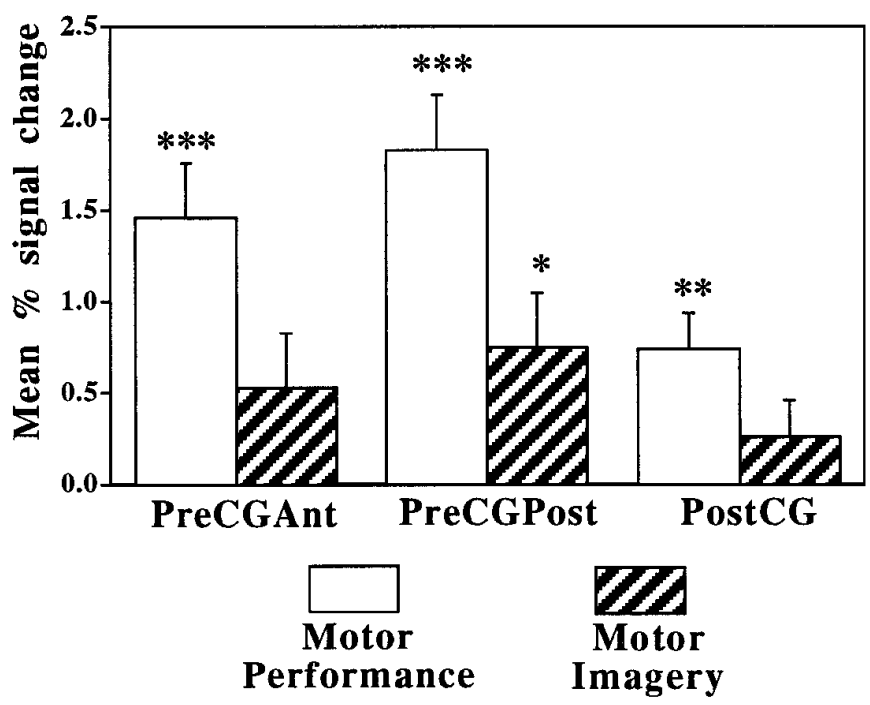

Figure 1. Histograms represent mean \pm SEM percentage fMRI signal changes in the anterior (PreCGAnt) and posterior (PreCGPost) portions of the precentral gyrus and in the postcentral gyrus (PostCG) during actual or imagined MP relative to mean values of the control (VI) condition. Significant differences from control values are $* p<0.05,{ }^{*} p<0.01$, and $* * * p<0.001$, respectively.

PreCGPost: $F=19.14, p<0.001$; PostCG: $F=7.66, p<0.01$ ). In PreCGPost, mean signal intensity values were significantly higher during the execution of the finger-tapping task $(t=5.03$, $p<0.001)$ than during the other two conditions, and they were higher during mental representation of the same motor sequence $(t=2.91, p<0.05)$ than during the VI task (Fig. 1). In PreCGAnt and PostCG, significant signal increases were found only during MP (PreCGAnt: $t=5.56, p<0.001$; PostCG: $t=3.35, p<0.01$ ), although in the PreCGAnt, a tendency toward an increase $(t=$ 2.07, $p<0.062$ ) was present during MI.

\section{Time profile of functional activation in different} pixel populations

The observed differences in mean fMRI signal intensities during MP and MI might be related to the presence of spatially segregated neuronal populations selectively activated during one task, to a differential involvement of overlapping neural networks during the two tasks, or to a combination of the two. In a preliminary analysis, pixels significantly $(r>0.7)$ activated during actual MP (population "MP") were identified by correlating the time course of their signal intensities over the 24 images acquired during VI or MP with a square-wave function. For each subject, the mean normalized signal intensities of the selected group of "MP" pixels lying in PreCGPost were then calculated for each of the 36 images (Fig. 2). A three-way, repeated-measures ANOVA (task $\times$ block $X$ image) confirmed that values were significantly affected by task $(F=100.8, p<0.001)$. Moreover, they were higher during MI $(t=7.72, p<0.001)$ than during VI and still higher during MP $(\mathrm{t}=10.3, p<0.001)$, suggesting that at least some movementrelated pixels were also engaged during mental representation of the same motor sequence.

To further test this hypothesis, activated pixels during MP, MI, or both conditions were identified by correlating their time profile of signal intensity over the 36 images with square-wave or doublestep waveforms (Fig. 3). Clusters of pixels, the signal time course of which was significantly $(r>0.7)$ correlated with a double-step function (Fig. $3 A$ ), were identified in the three ROIs in the 


\section{Precentral gyrus (posterior portion)}

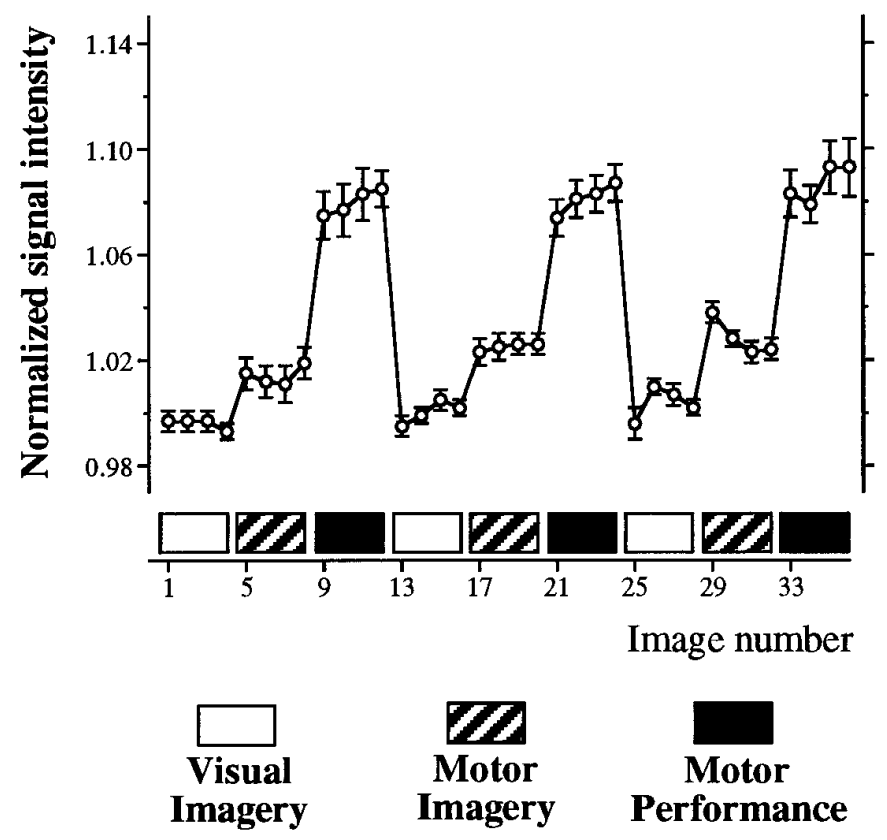

Figure 2. Time profile of mean normalized signal intensity in the population of pixels, located in the posterior portion of the precentral gyrus, displaying significant $(r>0.7)$ increases during actual MP (population MP). Each point represents mean \pm SEM values of one image in the series. The task sequence is shown at the bottom of the graph. A repeatedmeasures ANOVA performed on values of the 36 images showed that this pixel population was also significantly activated during MI (see Results).

majority of subjects (9/12 in PreCGAnt, 10/12 in PreCGPost, and $8 / 12$ in PostCG). To confirm that these pixel populations were indeed activated during both MP and MI, the mean normalized signal intensities of all identified "double-step" pixels (population "A") lying within each ROI were calculated for each subject and for each image, and data entered a four-way, repeated-measures ANOVA (region $\times$ task $\times$ block $\times$ image). Values were significantly different during the three tasks $(F=130.1, p<0.001)$, being higher during MI than VI $(t=8.09, p<0.001)$ and during MP relative to the other two conditions $(t=12.01, p<0.001)$. No significant difference was found in the time profile of signal intensity between the pixel populations of the three areas; mean percentage increases over control values during MI were $\sim 26 \%$ as great as the increases during MP in the PreCGPost (Fig. 4A), 32\% in PreCGAnt, and $33 \%$ in PostCG.

To assess whether other pixel populations were activated exclusively during MI or MP, two additional analyses were performed by comparing the time profile of signal intensity of each pixel in the three ROIs with waveforms $\mathrm{B}$ or $\mathrm{C}$ of Figure 3. Only one subject showed some pixels in the PreCGAnt and PreCGPost, the signal time course of which was selectively correlated with function $\mathrm{C}$ (that is, which displayed increased levels only during $\mathrm{MI}$ ). In approximately half of the subjects (6/12 in the PreCGAnt, 7/12 in PreCGPost, and 6/12 in PostCG), clusters of pixels were identified, the signal time course of which was selectively correlated with the waveform B in Figure 3 (population "B"). A 4-way, repeated-measures ANOVA showed that signal intensities of these pixels were affected by task $(F=37, p<0.001)$, whereas they did not differ significantly among the three ROIs. Values acquired during MI were indistinguishable from the control (VI) condition $(t=-0.17, p>0.85)$. By contrast, MP induced a clear-cut activation $(t=6.13, p<0.01)$. Thus, these pixels were indeed activated only during actual MP. The mean signal time course of population B pixels lying in the PreCGPost is shown in Figure $4 B$. It should be mentioned that some pixels were identified by (that is, the time course of their signal intensity was significantly correlated with) both waveforms A and B in Figure 3. Because repeated-measures ANOVA showed that their values acquired during MI were significantly higher than during VI, they were considered to belong to population A.

\section{Spatial distribution and extent of functional activation during $M I$ and $M P$}

Representative statistical maps obtained in two subjects, depicting the location and extent of populations $\mathrm{A}$ and $\mathrm{B}$, are shown in Figure 5. There was considerable intersubject variability, both in the anatomical conformation of the precentral and postcentral gyri and the extent and spatial distribution of the activated areas. Pixels activated by movement alone (population B) were intermingled with, or more often at the periphery of, clusters of pixels activated both during movement performance and imagery (population A). In the precentral gyrus, these populations could be found both in the depth of the banks of central and precentral sulci and in the gray matter at the convexity of the gyrus. More sparse activation was detectable in the postcentral gyrus (Fig. 5). No significant difference was found between the number of activated pixels or mean signal intensity changes in the deep (anterior
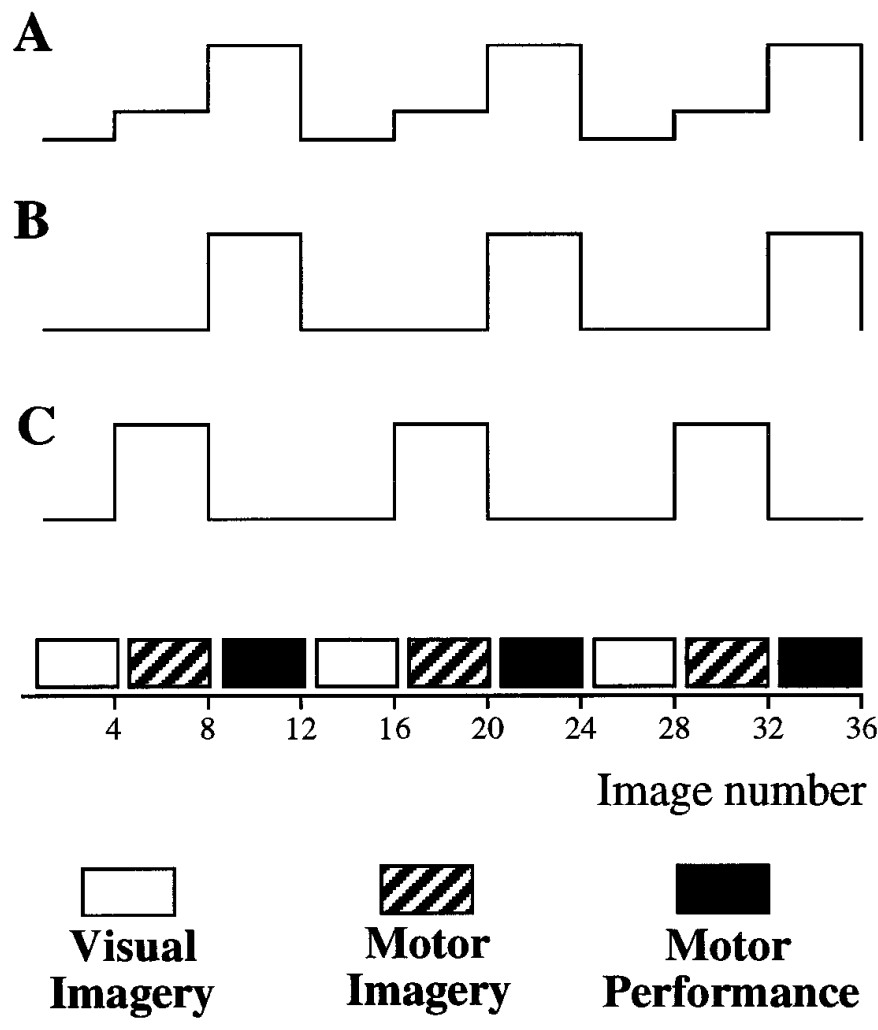

Figure 3. To identify pixels in the three ROIs significantly activated during MP, MI, or both conditions, their time profile of signal intensity was correlated with single-step $(B, C)$ or double-step $(A)$ waveforms. Based on data shown in Figures 1 and 2, the height of the first step in waveform A was set to $\sim 30 \%$ that of the second one. 


\section{Precentral gyrus (posterior portion)}

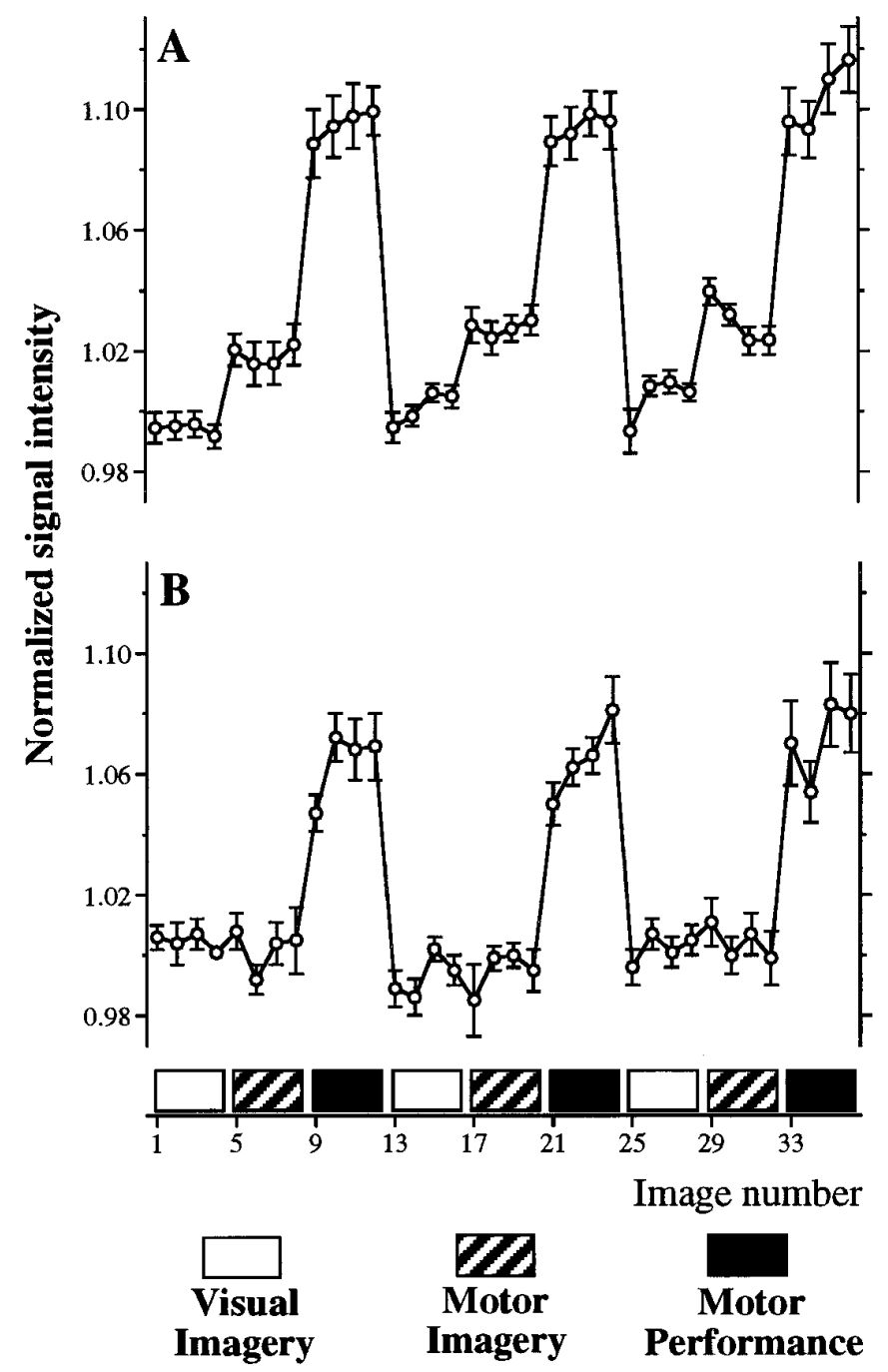

Figure 4. Time profile of normalized fMRI signal intensity in the pixel populations located in the posterior portion of the precentral gyrus, significantly activated during both actual MP and MI $(A)$ or MP alone $(B)$. Each point represents mean \pm SEM values of one image in the series. The task sequence is shown at the bottom of the graph.

bank of the central sulcus) and superficial (crown) portions of PreCGPost.

Mean activated volumes for population A and B were 131 and $15 \mathrm{~mm}^{3}$ in PreCGAnt, 164 and $36 \mathrm{~mm}^{3}$ in PreCGPost, and 117 and $36 \mathrm{~mm}^{3}$ in PostCG, respectively. If values are expressed as percentage fractions of the total (anatomical) volume of each ROI, it appears that the spatial extent of activation during MI and MP was much wider in the two precentral areas than in the postcentral area (Fig. 6). No significant correlation was found between the spatial extent or intensity of activation in any of the three ROIs and the degree of EMG activity displayed by the same subjects during MI (Fig. 7).

A final analysis was made to estimate whether the spatial distribution of pixels activated during both MI and MP (population A) was different from the whole population presumably activated by movement (population MP). All pixels belonging to population $\mathrm{A}$ in the three ROIs were also identified by the correlation analyses performed on the 24 images acquired during VI or MP (that is, population A corresponded to a fraction of population MP). Talairach coordinates (Table 1) of the centers of mass of populations A and of populations MP were calculated for each subject and compared by repeated-measures ANOVA. No significant difference was found between the two sets of values.

\section{Experiment 2}

In the previous group of experiments, the periods of mental representation of movement immediately preceded real motor acts. To test whether the observed signal increases during MI were attributable to, at least in part, an unintentional preparation to move, an additional series of experiments was performed in which the task order was MP-Rest-MI-VI. In this way, any increase of neural activity during MI attributable to a "preparatory" phenomenon should be cancelled, because it should conceivably be present also during the control task. The rest condition should also exclude possible after effects of MP on data acquired during MI.

Mean percentage changes of normalized signal intensities of all pixels lying in PreCGPost during MP, MI, or rest compared with the VI task are shown in Figure 8. A four-way, repeated-measures ANOVA (region $\times$ task $\times$ block $\times$ image) performed on values from the 48 images in the three ROIs revealed a significant effect of task $(F=44.03, p<0.001)$; mean signal intensities during MI were higher than during both VI and rest $(t=4.5, p<0.01)$, and values during MP were higher than during the other three conditions $(t=7.98, p<0.001)$.

To identify activated pixels during MP, MI, or both, crosscorrelation analyses were performed using the same single- or double-step reference waveforms used in the previous experiment (Fig. 3), adapted to the new task order. Population A pixels (Fig. $9 A$ ) meeting the statistical criterion were identified in eight of eight subjects in PreCGAnt and PreCGPost and in six of eight subjects in PostCG. Repeated-measures ANOVA showed that the time profile of their signal intensity was similar in the three ROIs, whereas it was significantly affected by task $(F=114.17 ; p<$ $0.001)$. Signal intensities during MI were higher than both VI and rest $(t=6.96, p<0.001)$, and still higher values were obtained during MP $(t=13.9, p<0.001)$. Mean percentage increases of signal intensities over the control (VI) values during MI were $\sim 28 \%$ as great as during MP in PreCGPost, $29 \%$ in PostCG, and $34 \%$ in PreCGAnt. The mean extent of the activated areas was $215 \mathrm{~mm}^{3}$ in PreCGPost, $208 \mathrm{~mm}^{3}$ in PostCG, and $140 \mathrm{~mm}^{3}$ in PreCGAnt.

Population B pixels (Fig. 9B) were found in three of eight volunteers in PreCGAnt, five of eight in PreCGPost, and six of eight in PostCG. Their signal time course was also affected by task $(F=20.32, p<0.01)$, with significant signal increases only during MP relative to the other three conditions $(t=8.89, p<0.01)$; similar values were found in the three ROIs. No pixel in any region was significantly activated only during $\mathrm{MI}$.

In the six subjects who participated in both experiments, there were no significant differences between the two studies in mean changes of signal intensity in the three ROIs during MI and MP (experiment: $F=1.58, p>0.25$; experiment $\times$ task: $F=2.55, p>$ 0.15 ; experiment $\times$ task $\times$ region: $F=0.04, p>0.85)$. The extent of population A in the three ROIs (experiment: $F=0.11, p>$ 0.75 ; experiment $\times$ region: $F=0.97, p>0.40)$ and the intensity of activation of the same population during MI and MP (experi- 
ment: $F=2.88, p>0.15$; experiment $\times$ task: $F=2.39, p>0.15$; experiment $\times$ task $\times$ region: $F=1.84, p>0.20$ ) were also similar in the two experiments. Accordingly, no significant difference in the extent and intensity of activation was found when comparing the results of the 12 studies of experiment 1 with those of the 8 studies of experiment 2 .
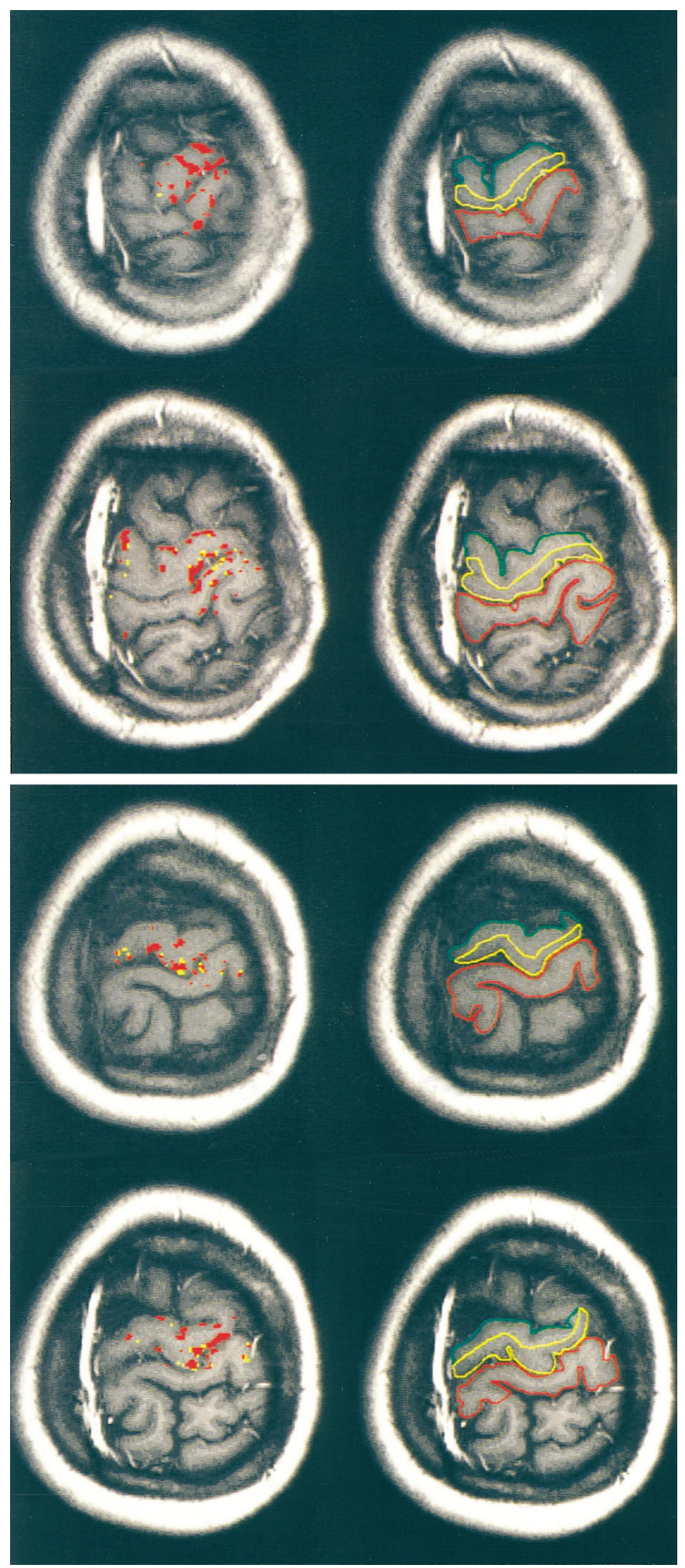
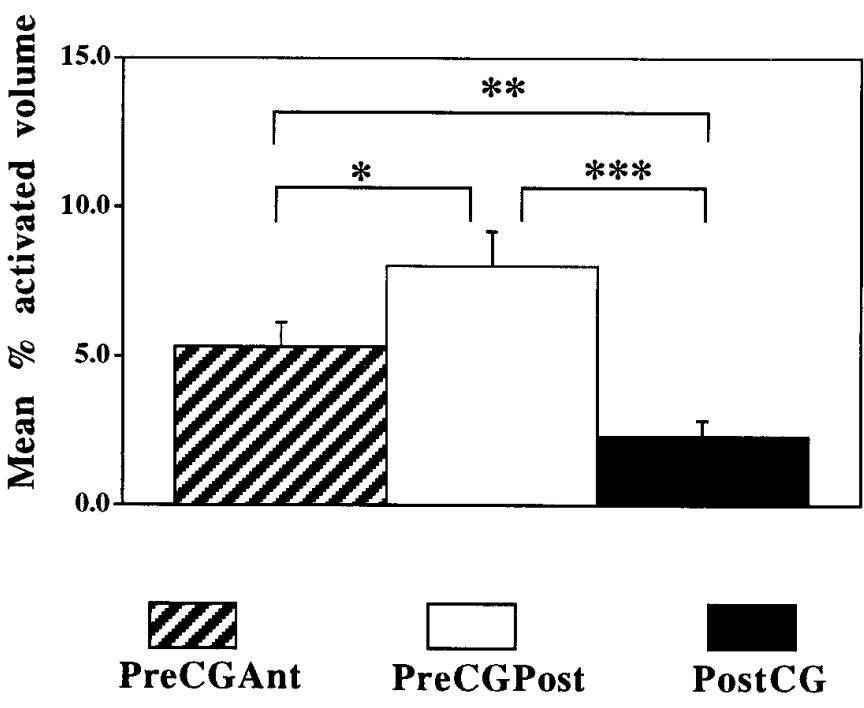

Figure 6. Histograms are mean \pm SEM of the activated volumes during both MP and MI (population A), expressed as percentage of the anatomical extent of the three ROIs. Significant differences at ${ }^{*} p<0.05,{ }^{* *} p<$ $0.01,{ }^{* * *} p<0.001$.

In comparing mean normalized signal intensity values obtained during VI, MI, and MP in the 20 experimental sessions, significant increases were found during MI (as well as during MP) in all three ROIs (PreCGAnt: $t=3.47, p<0.01$; PreCGPost: $t=4.67, p<0.001$; PostCG: $t=3.16, p<0.01)$. Mean percentage increases during MI and MP were $\sim 0.6$ and $1.7 \%$ in PreCGAnt, 0.8 and $2.1 \%$ in PreCGPost, and 0.4 and $1.2 \%$ in PostCG, respectively.

\section{DISCUSSION}

The results of this study indicate that functional activity levels in the posterior portion of the precentral gyrus, the presumed site of the primary motor cortex, are increased during mental representation of a simple sequence of finger movements, although the intensity of activation is lower than during real movements. Moreover, foci displaying significant activation during both MP and MI are present in the majority of subjects in the precentral and postcentral gyri, where they appear to represent a large fraction of the whole neural population activated by movement.

$\leftarrow$

Figure 5. Statistical maps, overlaid on $T_{1}$-weighted anatomical images of oblique planes along the left central sulcus, showing the location of activated points in the perirolandic cortex (left) and of the three identified ROIs (right) in two representative subjects. For each subject, two planes were studied, one covering the convexity of precentral and postcentral gyri (top) and the other the depth of central sulcus (bottom). In each image, top is anterior and right is lateral. Left, Pixels showing by correlation analysis significant fMRI signal increases during both actual MP and MI (population A) are indicated in red; pixels activated during MP alone (population B) in white-yellow. Right, Boundaries of the ROIs located in the postcentral gyrus (red), the posterior portion of the precentral gyrus (yellow), and the anterior portion of the precentral gyrus (green). The central sulcus corresponds to the limit between the red and yellow areas. The boundary between the two precentral areas is shown in yellow. Both areas in the precentral gyrus display clusters of pixels activated during both MP and MI and more sparse pixels activated only during actual MP. Discrete activated foci are also present in the postcentral gyrus, particularly in the subject displayed on top. 
Table 1. Talairach coordinates of the centers of mass of pixel populations activated during motor performance and motor imagery

Anteroposterior Lateral

Superioinferior

(y)

$(x)$

$(z)$

\begin{tabular}{llll}
\hline $\begin{array}{c}\text { Precentral gyrus } \\
(\text { anterior part })\end{array}$ & & & \\
MP $(n=12)$ & $-14.2 \pm 2.2$ & $29.4 \pm 1.4$ & $59.1 \pm 0.9$ \\
MP + MI $(n=9)$ & $-14.0 \pm 2.4$ & $29.5 \pm 1.4$ & $59.2 \pm 1.2$ \\
Precentral gyrus & & & \\
$\quad$ (posterior part) & & & \\
MP $(n=11)$ & $-18.6 \pm 1.8$ & $31.8 \pm 1.4$ & $57.0 \pm 0.8$ \\
MP + MI $(n=10)$ & $-18.2 \pm 1.8$ & $33.0 \pm 1.2$ & $56.7 \pm 1.0$ \\
Postcentral gyrus & & & \\
MP $(n=10)$ & $-24.7 \pm 2.6$ & $35.5 \pm 1.4$ & $56.6 \pm 0.8$ \\
MP + MI $(n=8)$ & $-24.4 \pm 3.0$ & $35.6 \pm 2.3$ & $57.0 \pm 1.0$
\end{tabular}

All data (mean \pm SEM, in $\mathrm{mm}$ ) refer to the left hemisphere, contralateral to the actual or imagined hand movements. Population MP includes all pixels activated during real movement, whereas population MP + MI (corresponding to population $\mathrm{A}$, see text) includes pixels activated during both actual movement and motor imagery. Only subjects displaying more than 4 activated pixels in each area were included in the analysis. $y$ values represent distances from the vertical plane passing through the anterior commissure (negative values are posterior to this plane). $x$ and $z$ values represent distances from the vertical and horizontal planes passing through the anterior and posterior commissures (positive values being left and superior), respectively.

\section{Gradient-echo fMRI mapping techniques: advantages and limitations}

fMRI provides a noninvasive tool for mapping human brain function (LeBihan and Karni, 1995). Specifically, $T_{2}{ }^{*}$ tissue relaxation-dependent proton signal increases can be detected by gradient-echo sequences on activation in specific brain regions, which are likely to reflect the interplay among local changes in blood flow, volume, and oxygenation (see Prichard and Rosen, 1994; Kwong, 1995). These fMRI signal changes are expected to be small, and the observed percentage increases in the three anatomically defined regions fit well with previous results on visual and motor cortex activation using similar fMRI techniques (Kwong et al., 1992; Frahm et al., 1993; Mattay et al., 1995).

The spatial resolution affordable by fMRI appears well suited to detect signal changes in discrete units of the human cortical gray matter (Frahm et al., 1993). However, because gradient-echo fMRI sequences are sensitive to inflow and large vessel effects, the effective spatial resolving power may be hampered if the observed signals are related predominantly to flow changes in large veins, draining blood from wide and potentially distant sites, rather than from the parenchimal capillary bed (Lai et al., 1993; Frahm et al., 1994; Segebarth et al., 1994). In the present study, pixels associated with visible blood vessels were excluded from the three ROIs. Moreover, the signal increases found in activated foci, on the order of $\leq 10 \%$, are likely to be induced by hemodynamic changes at the venule (vessel diameter, 50-200 $\mu \mathrm{m}$ ) (Haacke et al., 1994) and capillary levels and therefore to reflect local neural activity (see also Rostrup et al., 1995). Clear differences were indeed observed between the mean intensity and spatial extent of motorrelated activation in contiguous structures such as the anterior and posterior banks of the central sulcus, with higher values in motor areas.

\section{Experimental design}

To exclude potential effects of mental imagery per se, such as an aspecific arousal, in the present study, a VI task was adopted as the control condition. To the authors' knowledge, no study has so far described significant changes in functional activity levels in the primary sensorimotor area (SM1) during VI. Because subjects were asked to mentally scan the visual scene, some degree of neural activation related to actual or imagined eye movements otherwise might have been present (see Lang et al., 1994). The results of our second group of experiments show that fMRI signal intensity values during MI were higher than those during either VI or rest. Therefore, the observed increases during MI and MP are unlikely to be related to a downward shift of hemodynamic parameters during the control (VI) state.

Given the poor temporal resolution achieved in the present study (14 sec) compared with other fMRI techniques, fast hemodynamic changes could not be detected. It is well known that at the onset (or offset) of stimulation, fMRI signal intensities rise (decay) with a time constant of some seconds (Kwong et al., 1992; Bandettini et al., 1993; Friston et al., 1994). The adopted delay
Figure 7. Integrated EMG activity recorded from surface electrodes overlying the right thenar eminence (open circles) or medial forearm (triangles) plotted against the number of pixels in the PreCGPost showing a significant activation during both MI and MP; that is, the extent of population A (left) or the percentage changes of signal intensity during MI in the same pixel population (right). Multiple linear regression analyses yielded no significant correlation between the EMG values and neural activity (number of activated pixels: $r=$ $0.41 ; F=0.92 ; p>0.4$; intensity of activation: $r=0.15 ; F=0.10 ; p>0.9$ ). Similarly, no significant correlation was found between the EMG activity during MI and the spatial extent or intensity of activation of population A in the PreCGAnt (number of pixels: $r=0.46 ; F=$ $1.24 ; p>0.3$; intensity of activation: $r=$ $0.22 ; F=0.23 ; p>0.75)$, and the PostCG (number of pixels: $r=0.51 ; F=$ $1.58 ; p>0.25$; intensity of activation: $r=0.22 ; F=0.19 ; p>0.8)$.

\section{Precentral gyrus (posterior portion)}
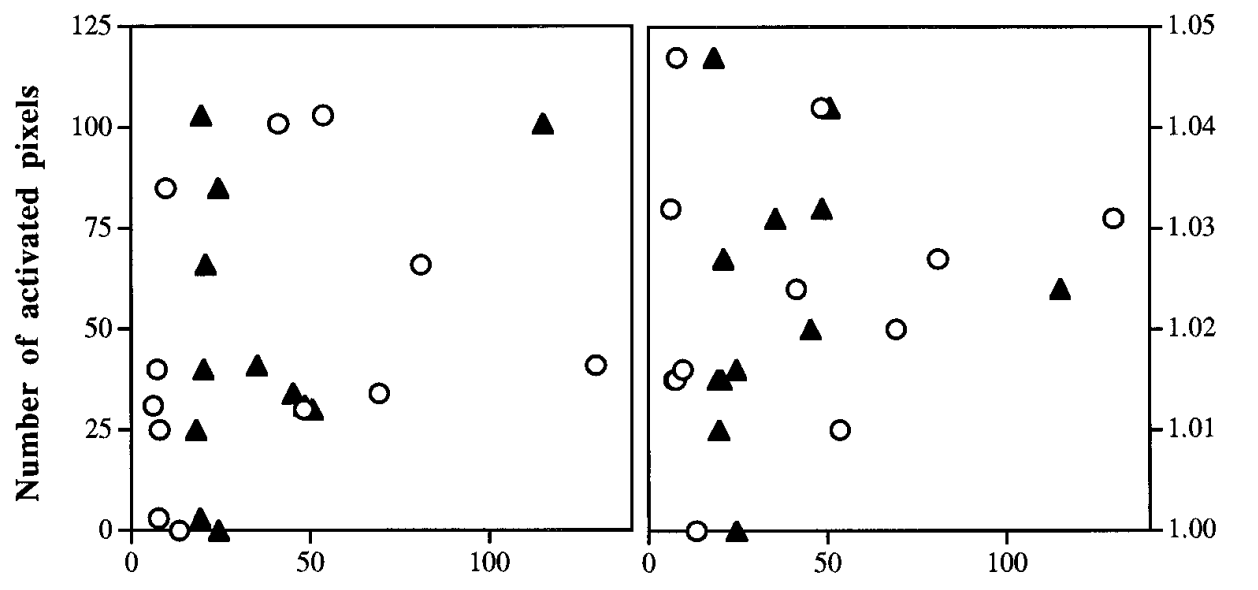

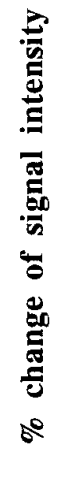



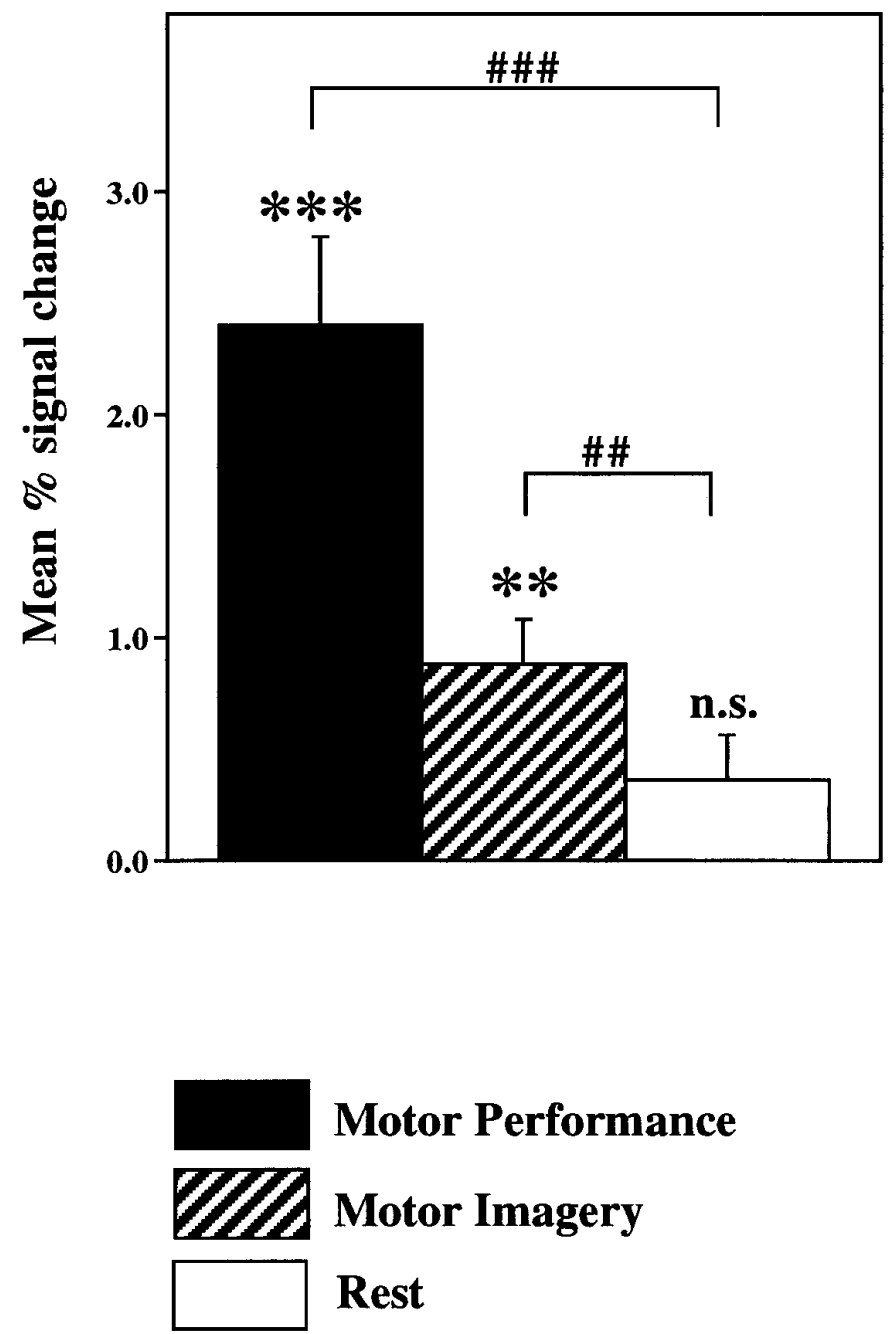

Figure 8. Mean \pm SEM percentage fMRI signal changes in the PreCGPost during actual or imagined MP or rest, relative to mean values of the control (VI) condition, in the eight subjects participating in experiment 2. Significant differences from values of the VI task at $* * p<0.01$ and ${ }^{* * *} p<$ 0.001 , respectively; n.s., not significant. Significant differences at ${ }^{\# \# p}<<$ 0.01 and ${ }^{\# \# \#} p<0.001$, respectively.

between the beginning of each task and image acquisition should exclude major after effects. In any case, because MI was never immediately preceded by actual MP, the observed signal changes during mental representation of motor acts cannot be attributed to residual effects of the latter condition. On the other hand, similar results were obtained when MI immediately preceded actual MP (experiment 1) or the control condition (experiment 2). Therefore, fMRI signal changes during MI were not simply attributable to an unintentional preparation to move.

\section{Movement-related functional activation in the perirolandic area}

The cortical primary motor area has long been associated with the control of distal arm movements (for review, see Porter and Lemon, 1993), and motor tasks involving repetitive finger flexion have been consistently shown to increase functional activity levels in the contralateral SM1 cortex in humans (Roland et al., 1980; Fox et al., 1987; Colebatch et al., 1991; Kim et al., 1993; Matelli et al., 1993; Rao et al., 1993, 1995; Shibasaki et al., 1993; Dettmers et al., 1995). The present results showed that during a self-paced

\section{Precentral gyrus (posterior portion)}
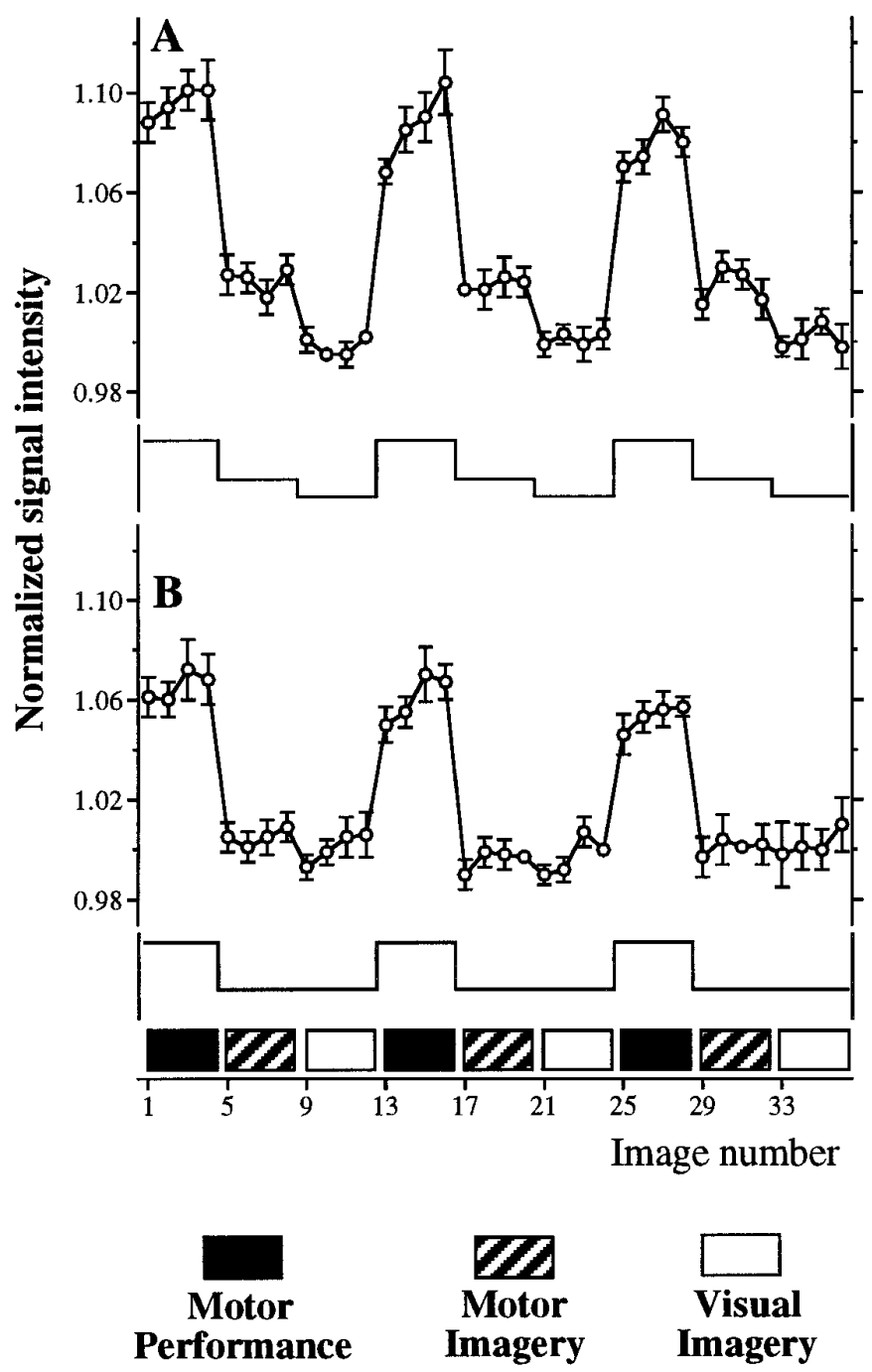

Figure 9. Time profile of normalized fMRI signal intensity in the pixel populations located in the posterior portion of the precentral gyrus, significantly activated during both actual MP and MI $(A)$ or MP alone $(B)$, in experiments in which MI preceded the control (VI) task. Each point represents mean \pm SEM $(n=8)$ values of one image in the series. The reference waveforms and the task sequence are shown at the bottom of the graphs.

finger-tapping task, the spatial extent and mean intensity of functional activity changes within the sampled portion of SM1 were significantly higher in the two precentral regions, including the primary motor cortex and the adjacent portion of the lateral premotor cortex, than in the postcentral gyrus. These findings are likely to reflect the more direct involvement of precentral areas in motor control. In all subjects, multiple activated foci were identified in the precentral gyrus, probably representing overlapping areas controlling distal movements (Rao et al., 1995; Sanes et al., 1995).

\section{MP and MI: common neural substrates?}

In the present study, significant increases in functional activity levels at the presumed site of the primary motor cortex were 
found during MI, which were not evident or could be detected only in a few subjects, in previous functional imaging mapping investigations (see introductory remarks). It is likely that several factors contribute to this apparent discrepancy. Levels of activation during imagery were relatively low compared with actual MP; therefore, they may have been undetected by imaging techniques characterized by lesser spatial resolution, particularly when activity levels from the precentral and postcentral areas could not be clearly separated. Moreover, given the complexity of motor subsystems, quantitative differences in the pattern of activation of motor and premotor areas are to be expected when different kinds of motor acts are executed or imagined (see Passingham, 1993; Ashe and Ugurbil, 1994; Jeannerod et al., 1995). For instance, mental rehearsal of visually guided grasping movements increases blood flow rates in inferior lateral premotor regions (Decety et al., 1994), whereas the supplementary motor area appears to be more active during mental representation of other kinds of motor tasks (Roland et al., 1980; Rao et al., 1993; Stephan et al., 1995). In the present study, a simple and predictable self-paced sequence of finger movements was used, which can be easily implemented, even without a training period. It can be speculated that the neural networks organizing simple, "unlearned" sequences of finger movements are also distributed in the precentral areas, which have been recently suggested to participate in the organization of complex motor acts in humans (Shibasaki et al., 1993). There is indeed evidence that neuronal assemblies in the primate motor cortex are related to complex, cognitive processes (Georgopoulos et al., 1993). For instance, directionally tuned cells in the primary motor area discharge during the delay period (without concomitant changes in EMG activity) in delayed (Tanji and Evarts, 1976; Georgopoulos et al., 1989a; Alexander and Crutcher, 1990) or memorized (Smyrnis et al., 1992) motor tasks. Moreover, time-related changes in the activity of directionally selective neurons may encode mental rotation of the direction of intended movement (Georgopoulos et al., 1989b). It may be hypothesized that the activity of similar networks, lying in the precentral gyrus, might be accessible to conscious inspection in humans, and thus be involved in MI. When more complex, learned sequences of motor acts must be retrieved, the supplementary motor area is more likely to be involved (Roland et al., 1980; Rao et al., 1993).

Local hemodynamic changes during brain activation are thought to reflect primarily alterations in synaptic activity, which may in turn be attributable to increased firing in local interneurons (either excitatory or inhibitory) and/or in afferent fibers (Raichle, 1987; Roland, 1993). In the investigated regions, foci activated during MI were always active (and displayed higher signal increases) during MP as well, suggesting that imagery involves at least a subset of neurons that are engaged during actual MP. It cannot be fully established whether the observed fMRI signal increases during mental representation of motor acts are attributable entirely to the activity of intracortical networks or whether they are related in part to motor or sensory feedback signals attributable to increased descending volleys. A related issue concerns the mechanisms and sites (cortical or spinal) of motor inhibition during MI (see Jeannerod, 1994; Berthoz, 1996; Lang et al., 1996). There are several reports of an increase of EMG activity in muscles involved in the imagined motor act (Jacobson, 1930; Wehner et al., 1984; Harris and Robinson, 1986), although this is not a generalized finding (Yue and Cole, 1992) (for discussion, see Jeannerod, 1994). In the present study, no subject displayed overt motor behavior during MI, and only some showed a small increase of EMG activity during MI compared with the control condition. Although it is, of course, possible that the activity of muscles other than that recorded could have been changed, regression analyses showed that cortical activation was not related to the degree of EMG activity. Thus, it is conceivable that the observed fMRI changes during MI are attributable primarily to the activity of intracortical circuits.

It is intriguing that in the majority of subjects, an increase in functional activity levels was found not only during MP but also during MI in discrete neural units lying in the postcentral gyrus. Recent results suggest that the primary somatosensory area displays higher levels of neural activity during imagery of tactile stimuli (Hodge et al., 1996). In the present study, subjects were asked to recall during MI the sensations associated with fingertapping, which are both exteroceptive and proprioceptive in nature. Thus, it might be speculated that the observed fMRI changes in the postcentral gyrus during $\mathrm{MI}$ are related, at least in part, to somatosensory imagery.

The present fMRI results are in line with recent studies suggesting an involvement of the primary motor area during MI. Imagination of hand movements induced specific changes in the pattern of DC potentials recorded from sites overlying the primary sensorimotor cortex (Beisteiner et al., 1995) and in the pattern of magnetic fields related to activity of the primary motor cortex (Lang et al., 1996), although in both studies, changes were less pronounced than those induced by actual MP. In a transcranial magnetic stimulation study, PascualLeone et al. (1995) showed that after $5 \mathrm{~d}$ of mental practice of a five-finger piano exercise, the cortical motor output maps targeting finger flexors and extensors enlarged, and their activation threshold decreased. Sirigu et al. (1995) described a patient with a focal lesion of the right motor cortex, who displayed congruent and selective unilateral impairments of motor behavior and MI of the contralateral hand, as judged by the actual and perceived time required to perform overt or covert movements, respectively.

Altogether, these observations suggest that primary motor cortex plays a role in the mental representation of motor acts. Furthermore, the present results show that conscious representation of movement induces changes in activity of neural networks in the perirolandic cortical area, largely overlapping the changes involved in motor execution.

\section{REFERENCES}

Alexander GA, Crutcher MD (1990) Preparation for movement: neural representations of intended direction in three motor areas of the monkey. J Neurophysiol 64:133-150.

Ashe J, Ugurbil K (1994) Functional imaging of the motor system. Curr Opin Neurobiol 4:832-839.

Bandettini PA, Jesmanowicz A, Wong EC, Hyde JS (1993) Processing strategies for time-course data sets in functional MRI of the human brain. Magn Reson Med 30:161-173.

Beisteiner R, Höllinger P, Lindinger G, Lang W, Berthoz A (1995) Mental representations of movements. Brain potentials associated with imagination of hand movements. Electroencephalogr Clin Neurophysiol 96:183-193.

Berthoz A (1996) The role of inhibition in the hierarchical gating of executed and imagined movements. Cognit Brain Res 3:101-113.

Colebatch JG, Deiber MP, Passingham RE, Friston KJ, Frackowiak RSJ (1991) Regional cerebral blood flow during voluntary arm and hand movements in human subjects. J Neurophysiol 65:1392-1401.

Decety J, Sjoholm H, Ryding E, Sternberg G, Ingvar D (1990) The cerebellum participates in cognitive activity: tomographic measurements of regional cerebral blood flow. Brain Res 535:313-317. 
Decety J, Jeannerod M, Germain M, Pastene J (1991) Vegetative response during imagined movement is proportional to mental effort. Behav Brain Res 42:1-5.

Decety J, Perani D, Jeannerod M, Bettinardi V, Tadary B, Woods R, Mazziotta JC, Fazio F (1994) Mapping motor representations with positron emission tomography. Nature 371:600-602.

Dettmers C, Fink GR, Lemon RN, Stephan KM, Passingham RE, Silbersweig D, Holmes A, Ridding MC, Brooks DJ, Frackowiak RSJ (1995) Relation between cerebral activity and force in the motor areas of the human brain. J Neurophysiol 74:802-815.

Fox PT, Pardo JV, Petersen SE, Raichle ME (1987) Supplementary motor and premotor responses to actual and imagined hand movements with positron emission tomography. Soc Neurosci Abstr 13:1433.

Frahm J, Merboldt KD, Hänicke W (1993) Functional MRI of human brain activation at high spatial resolution. Magn Reson Med 29:139-144.

Frahm J, Merboldt KD, Hänicke W, Kleinschmidt A, Boecker H (1994) Brain or vein-oxygenation or flow: on signal physiology in functional MRI of human brain activation. NMR Biomed 7:45-53.

Friston KJ, Jezzard P, Turner R (1994) Analysis of functional MRI time-series. Hum Brain Mapp 1:153-171.

Georgopoulos AP, Crutcher MD, Schwartz AB (1989a) Cognitive spatial-motor processes. III. Motor cortical prediction of movement direction during an instructed delay period. Exp Brain Res 75:183-194.

Georgopoulos AP, Lurito JT, Petrides M, Schwartz AB, Massey JT (1989b) Mental rotation of the neuronal population vector. Science 243:234-236.

Georgopoulos AP, Taira M, Lukashin A (1993) Cognitive neurophysiology of the motor cortex. Science 260:47-52.

Geyer S, Zilles K, Simon U, Schormann T, Dabringhaus A, Schleicher A, Roland PE (1995) Architectonic and receptor autoradiographic mapping of the human primary motor cortex. Hum Brain Mapp [Suppl 1]:290.

Grafton ST, Woods RP, Mazziotta JC, Phelps ME (1991) Somatotopic mapping of the primary motor cortex in humans: activation studies with cerebral blood flow and positron emission tomography. J Neurophysiol 66:735-743.

Grafton ST, Woods RP, Mazziotta JC (1993) Within-arm somatotopy in human motor areas determined by positron emission tomography imaging of cerebral blood flow. Exp Brain Res 95:172-176.

Haacke EM, Hopkins AL, Lai S, Buckley P, Friedman L, Meltzer H, Hedera P, Friedland R, Klein S, Thompson L, Detterman D, Tkach J, Lewin JS (1994) 2D and 3D high-resolution gradient-echo functional imaging of the brain: venous contributions to signal in motor cortex studies. NMR Biomed 7:54-62.

Harris KS, Robinson WJ (1986) The effect of skill level on EMG activity during internal and external imagery. J Sport Psychol 8:105-111.

Hodge C, Dubroff J, Huckins S, Szeverenyi N (1996) Somatosensory imagery activates primary sensory cortex in human: a functional MRI study. Neuroimage 3:S209.

Ingvar DH, Philipson L (1977) Distribution of cerebral blood flow in the dominant hemisphere during motor ideation and motor performance. Ann Neurol 2:230-237.

Irani M, Peleg S (1991) Improving resolution by image registration. Comput Vis Graphics Image Processing 53:231-239.

Jacobson E (1930) Electrical measurements of neuromuscular states during mental activities. I. Imagination of movement involving skeletal muscle. Am J Physiol 91:567-608.

Jeannerod M (1994) The representing brain: neural correlates of motor intention and imagery. Behav Brain Sci 17:187-245.

Jeannerod M, Arbib MA, Rizzolatti G, Sakata H (1995) Grasping objects: the cortical mechanisms of visuomotor transformation. Trends Neurosci 18:314-320.

Kim S-G, Ashe J, Georgopoulos AP, Merkle H, Ellermann JM, Menon RS, Ogawa S, Ugurbil K (1993) Functional imaging of human motor cortex at high magnetic field. J Neurophysiol 69:297-302.

Kwong KK (1995) Functional magnetic resonance imaging with echo planar imaging. Magn Reson Q 11:1-13.

Kwong KK, Belliveau JW, Chesler DA, Golberg IE, Weisskoff RM, Poncelet BP, Kennedy DN, Hoppel BE, Cohen MS, Turner R, Cheng H-M, Brady TJ, Rosen BR (1992) Dynamic magnetic resonance imaging of human brain activity during primary sensory stimulation. Proc Natl Acad Sci USA 89:5675-5679.

Lai S, Hopkins AL, Haacke EM, Li D, Wasserman BA, Buckley P, Friedman L, Meltzer H, Hedera P, Friedland R (1993) Identification of vascular structures as a major source of signal contrast in high resolution $2 \mathrm{D}$ and $3 \mathrm{D}$ functional activation imaging of the motor cortex at 1.5T: preliminary results. Magn Reson Med 30:387-392.

Lang W, Petit L, Höllinger P, Pietrzyk U, Tzourio N, Mazoyer B, Berthoz A (1994) A positron emission tomography study of oculomotor imagery. NeuroReport 5:921-924.

Lang W, Cheyne D, Höllinger P, Gerschlager W, Lindinger G (1996) Electric and magnetic fields of the brain accompanying internal simulation of movement. Cognit Brain Res 3:125-129.

Le Bihan D, Karni A (1995) Applications of magnetic resonance imaging to the study of human brain function. Curr Opin Neurobiol 5:231-237.

Leonardo M, Fieldman J, Sadato N, Campbell G, Ibañez V, Cohen L, Deiber M-P, Jezzard P, Pons T, Turner R, Le Bihan D, Hallett M (1995) A functional magnetic resonance imaging study of cortical regions associated with motor task execution and motor ideation in humans. Hum Brain Mapp 3:83-92.

Mahoney MJ, Avener M (1987) Psychology of the elite athlete. An explorative study. Cognit Ther Res 1:135-141.

Matelli M, Rizzolatti G, Bettinardi V, Gilardi MC, Perani D, Rizzo G, Fazio F (1993) Activation of precentral and mesial motor areas during the execution of elementary proximal and distal arm movements: a PET study. NeuroReport 4:1295-1298.

Mattay VS, Weinberger DR, Barrios FA, Sobering GS, Kotrla KJ, van Gelderen P, Duyn JH, Sexton RH, Moonen CTW, Frank JA (1995) Brain mapping with functional MR imaging: comparison of gradientecho-based exogenous and endogenous contrast techniques. Radiology 194:687-691.

Oldfield RC (1971) The assessment and analysis of handedness: the Edinburgh inventory. Neuropsychologia 9:97-113.

Parsons LM, Fox PT, Downs JH, Glass T, Hirsch TB, Martin CC, Jerabek PA, Lancaster JL (1995) Use of implicit motor imagery for visual shape discrimination as revealed by PET. Nature 375:54-58.

Pascual-Leone A, Dang N, Cohen LG, Brasil-Neto JP, Cammarota A, Hallett M (1995) Modulation of muscle responses evoked by transcranial magnetic stimulation during the acquisition of new fine motor skills. J Neurophysiol 74:1037-1045.

Passingham R (1993) The frontal lobes and voluntary action. Oxford: Oxford UP.

Porter R, Lemon R (1993) Corticospinal function and voluntary movement. Oxford: Oxford Science.

Prichard JW, Rosen BR (1994) State of the art review: functional study of the brain by NMR. J Cereb Blood Flow Metab 14:365-372.

Raichle ME (1987) Circulatory and metabolic correlates of brain function in normal humans. In: Handbook of physiology, Sec 1, The nervous system, Vol 5, Higher functions of the brain (Plum F, ed), pp 643-674. New York: Oxford UP.

Rao SM, Binder JR, Bandettini PA, Hammeke TA, Yetkin FZ, Jesmanowicz A, Lisk LM, Morris GL, Mueller WM, Estkowski LD, Wong EC, Haughton VM, Hyde JS (1993) Functional magnetic resonance imaging of complex human movements. Neurology 43:2311-2318.

Rao SM, Binder JR, Hammeke TA, Bandettini PA, Bobholz JA, Frost JA, Myklebust BM, Jacobson RD, Hyde JS (1995) Somatotopic mapping of the human primary motor cortex with functional magnetic resonance imaging. Neurology 45:919-924.

Roland PE (1993) Brain activation. New York: Wiley.

Roland PE, Zilles K (1994) Brain atlases: a new research tool. Trends Neurosci 17:458-467.

Roland PE, Larsen B, Lassen NA, Skinhøj E (1980) Supplementary motor area and other cortical areas in organization of voluntary movements in man. J Neurophysiol 43:118-136.

Rostrup E, Larsson HBW, Toft PB, Garde K, Henriksen O (1995) Signal changes in gradient-echo images of human brain induced by hypoxia and hyperoxia. NMR Biomed 8:41-47.

Rumeau C, Tzourio N, Peretti-Viton P, Levrier O, Joliot M, Mazoyer B, Salamon G (1994) Location of hand function in the sensorimotor cortex: MR and functional correlation. AJNR Am J Neuroradiol 15:567-572.

Sabbah P, Simond G, Levrier O, Habib M, Trabaud V, Murayama N, Mazoyer BM, Briant JF, Raybaud C, Salamon G (1995) Functional magnetic resonance imaging at $1.5 \mathrm{~T}$ during sensory motor and cognitive tasks. Eur Neurol 35:131-136.

Sanes JN, Stern CE, Baker JR, Kwong KK, Donoghue JP, Rosen BR (1993) Human frontal motor cortical areas related to motor performance and mental imagery. Soc Neurosci Abstr 18:1208. 
Sanes JN, Donoghue JP, Thangaraj V, Edelman RR, Warach S (1995) Shared neural substrates controlling hand movements in human motor cortex. Science 268:1775-1777.

Segebarth C, Belle V, Delon C, Massarelli R, Decety J, Le Bas J-F, Décorps M, Benabid AL (1994) Functional MRI of the human brain: predominance of signals from extracerebral veins. NeuroReport 5:813-816.

Shibasaki H, Sadato N, Lyshkow H, Yonekura Y, Honda M, Nagamine T, Suwazono S, Magata Y, Ikeda A, Miyazaki M, Fukuyama H, Asato R, Konishi J (1993) Both primary motor cortex and supplementary motor cortex play an important role in complex finger movement. Brain 116:1387-1398.

Sirigu A, Cohen L, Duhamel JR, Pillon B, Dubois B, Agid Y, PierrotDeseilligny C (1995) Congruent unilateral impairments for real and imagined hand movements. NeuroReport 6:997-1001.

Smyrnis N, Taira M, Ashe J, Georgopoulos AP (1992) Motor cortical activity in a memorized delay task. Exp Brain Res 92:139-151.

Stephan KM, Fink GR, Passingham RE, Silbersweig D, CeballosBaumann AO, Frith CD, Frackowiak RSJ (1995) Functional anatomy of the mental representation of upper extremity movements in healthy subjects. J Neurophysiol 73:373-386.

Talairach J, Tournoux P (1988) Co-planar stereotaxic atlas of the human brain. Stuttgart: Thieme.

Tanji I, Evarts EV (1976) Anticipatory activity of motor cortex neurons in relation to direction of an intended movement. J Neurophysiol 39:1062-1068.

Wang Y, Morgan WP (1992) The effects of imagery perspectives on the physiological responses to imagined exercise. Behav Brain Res 52:167-174.

Wehner T, Vogt S, Stadler M (1984) Task-specific EMG characteristics during mental practice. Psychol Res 46:369-401.

Woods RP, Cherry SR, Mazziotta JC (1992) Rapid automated algorithm for aligning and reslicing PET images. J Comput Assist Tomogr 115:565-587.

Yue G, Cole KJ (1992) Strength increases from the motor program: comparison of training with maximal voluntary and imagined muscle contractions. J Neurophysiol 67:1114-1123. 